\title{
Penile dermatoses: a clinical and histopathological study
}

\author{
R J Hillman, M M Walker, J R W Harris, D Taylor-Robinson
}

\begin{abstract}
Objective-To assess the spectrum of genital dermatological conditions affecting men and compare the clinical and histopathological diagnoses.

Design-Prospective study over a one year period.

Setting-A central London teaching hospital.

Patients-Seventy one patients with unresponsive penile dermatoses attending a specific internal referral clinic within the department of genitourinary medicine and 36 patients undergoing penile biopsy following attendance at other departments within the same hospital.

Methods-Full dermatological assessment of patients attending the specific clinic. Standard histopathological methods were used in the diagnosis of biopsy specimens.
\end{abstract} cal diagnosis of cutaneous penile abnormalities.

Results-Description of the range and relative frequency of penile dermatological conditions. The most common histopathological diagnosis was of non specific dermatitis. Twenty seven percent (16 of 61) of patients attending the specific clinic and 33\% (12 of 36) of men attending other departments had conditions requiring long term follow up.

Conclusions-The ranges of penile dermatoses presenting to the different departments were broadly similar. Penile biopsy was shown to be a safe and clinically informative procedure. In the genitourinary clinic setting, clinical diagnosis prior to biopsy was found frequently to be inaccurate.

Transmitted Diseases, Clinical Research Centre, Watford Road, Harrow, Middlesex, HA1 3UJ

R J Hillman

D Taylor-Robinson

St Mary's Hospital, London, UK.

Department of

Histopathology

M M Walker

Department of

Genitourinary

Medicine

J R W Harris

Address correspondence to:

Dr R J Hillman, The Jefferiss

Wing, St Mary's Hospital,

Praed Street, London W2

INY, UK.

Accepted for publication

5 March 1992

\section{Introduction}

Penile dermatoses encountered in genitourinary medicine (GUM) clinics frequently provide diagnostic difficulties. The diagnosis and treatment of such conditions is often empirical, based on the assumption that the rash is likely to be either fungal or eczematous. Not only is this approach unscientific, but it often fails to resolve, and in some cases, may exacerbate the problem. Patients with such dermatoses may persistently attend with relapsing rashes of uncertain aetiology, and potentially serious conditions can be overlooked. Presentation to other specialties such as general practice, urology, dermatology and general surgery also
Outcome measured-Clinico-pathologi-

occurs and such departments may lack the resources to diagnose accurately the wide range of sexually transmitted and other diseases affecting the penis.

The true nature and extent of penile dermatological conditions presenting to GUM clinics is unknown. In view of this, we investigated patients with persistent penile dermatoses by careful clinical evaluation, including biopsy. In addition, histopathological results from the specific referral clinic were compared with those of similar specimens submitted to the histopathology department over the same period from other departments.

\section{Methods}

A specific internal referral clinic, the Penile Dermatosis Clinic (PDC), was set up within the GUM department for men with persistent penile dermatoses which did not respond to treatment, or of which the diagnosis was in doubt and the clinician felt that biopsy would be helpful in establishing the diagnosis. All patients were screened for the commonly occurring sexually transmitted pathogens, and treatment was given where necessary. Patients were asked to avoid any specific local treatment to the area for a minimum of two weeks prior to assessment.

During the 45 minutes allocated for each patient, a full history, particularly including that of any personal or family dermatological conditions, was combined with a thorough examination of skin and mucous membranes, together with penoscopy. If a diagnosis could not be made at that point, or significant pathological changes could not be reasonably excluded on clinical grounds, the patient was counselled and offered a biopsy examination.

Under aseptic conditions the area to be biopsied, which included, where possible, the edge of the lesion and normal skin, was infiltrated with 1 to $2 \mathrm{ml}$ of $2 \%$ lignocaine with 1:200,000 adrenalin. Once anaesthesia had been achieved, the skin was gently pinched up using toothed forceps and an ellipse of approximately $\mathrm{lmm}$ by $2 \mathrm{~mm}$ was removed using scissors (fig). The biopsy specimen was then placed in $10 \%$ formal saline, processed routinely and stained by haematoxylin and eosin for histological examination. Haemostasis was achieved using silver nitrate sticks. In view of the small biopsy site, primary closure was not necessary, and the patient was given simple hygiene advice.

Samples from patients attending other specialties were received by the histopathology 


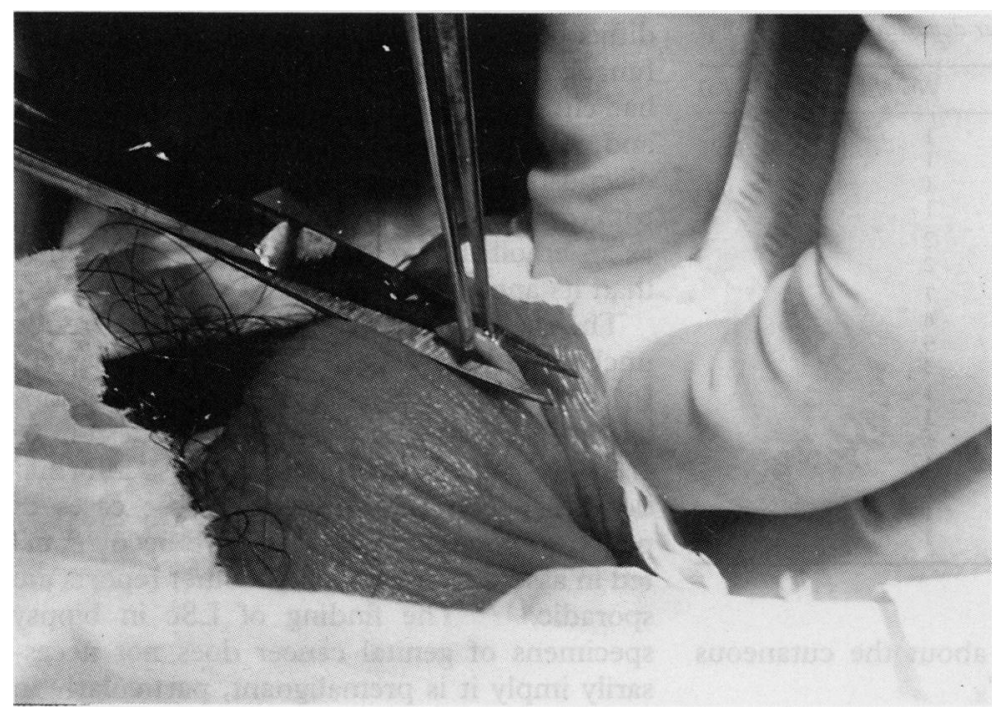

Figure Skin snip biopsy procedure

department in the course of routine clinical practice, without the patient being referred to the PDC. No patients were seen in more than one department.

Standard criteria were observed for the diagnosis of well recognised dermatological conditions, for example lichen sclerosus, lichen planus and dermatitis.' Previously established histopathological criteria for the diagnosis of wart virus infection and penile intraepithelial neoplasia (PIN) were applied. ${ }^{2}$ All specimens in which a histological diagnosis of nonspecific dermatitis (NSD) was made were additionally stained by the periodic acid Schiff method.

The initial clinical diagnosis was made prior to biopsy and compared with the final diag-

Table 1 Clinical diagnoses in patients not undergoing penile biopsy (one patient in each category)

Clinically apparent diagnoses

Fungal infection

Wart virus infection

Folliculitis

Herpes simplex virus infection

Atopic eczema

Pearly penile papules

Pearly penile papules
Penile vein thrombosis

Penile vein
Vitiligo

Psychiatric illness

Biopsy declined by patient

Wart virus infection

Atopic eczema

Table 2 Diagnoses in patients undergoing penile biopsy in the GUM department

\begin{tabular}{llc}
\hline Category & Diagnosis & No. of Patients $n=60$ \\
\hline Infection & Fungal infection & 1 \\
& Wart virus infection & $14(23 \%)$ \\
& Wart virus infection + dysplasia & 1 \\
Dermatosis & Non specific dermatitis & $16(26 \%)$ \\
& Lichen sclerosus & $9(15 \%)$ \\
& Lichen planus & $4(7 \%)$ \\
& Lichen simplex & 1 \\
& Psoriasis & $2(3 \%)$ \\
Neoplasia & Dermatitis artefacta & 1 \\
& PIN 2 & $2(3 \%)$ \\
& PIN 3 & $2(3 \%)$ \\
Miscellaneous & Basal cell papilloma & 1 \\
& Not diagnostic & $2(3 \%)$ \\
& Scarring & 1 \\
& Granulomatous disease ? cause & 1 \\
& Granuloma annulare & 1 \\
& Plasma cell balanitis & 1 \\
\hline
\end{tabular}

nosis which was based on the clinical features and histological findings. Thus, an essential part of the evaluation of the patient was discussion between the clinician and the histopathologist.

\section{Results}

Patients seen in the PDC

Seventy-one patients were seen in the PDC over a one year period. Seven $(10 \%)$ of the patients were homosexual and one of them was known to be infected with the human immunodeficiency virus. The age range of all patients was $18 \cdot 3$ to $65 \cdot 1$ years (average $36 \cdot 2$ years). Sixty patients underwent biopsy, two declined to have biopsies and nine had clinical conditions for which biopsy was not considered necessary (table 1 ). The age range of those undergoing penile biopsy was 22.5 to $65 \cdot 1$ years (average 36.0 years). The age range of those not having a biopsy was 18.3 to $56 \cdot 2$ years (average 37.3 years).

A short course of antibiotics for secondary infection was required by three $(5 \%)$ of the 60 patients undergoing biopsy, otherwise recovery was satisfactory, with minimal or no scarring, and return to full sexual activity usually within two weeks.

The diagnoses based on biopsy examination are shown in table 2 .

Histological findings were consistent with the initial clinical diagnosis in 20 (33\%) of the 60 patients undergoing biopsy. Lichen sclerosus (LSc) was diagnosed accurately prior to biopsy in seven $(78 \%)$ of nine cases, wart virus infection in $7(50 \%)$ of 14 cases, non specific dermatosis in five (31\%) of 16 cases and lichen planus in one $(25 \%)$ of four cases. Four $(7 \%)$ of the biopsies undertaken in the PDC showed evidence of PIN, which had not been suspected prior to biopsy.

\section{Patients seen in other departments}

During the same period, the histopathology department received penile biopsy specimens from 36 patients attending other departments within the hospital, comprising 28 from the urologists and four each from the dermatologists and the general surgeons. The age range of these patients was 16.2 to 81.2 years (average 46.4 years).

Less clinical information was available on these patients. In view of this, clinical concordance was not assessed and the histological diagnoses (table 3) were used only to define the spectrum of penile dermatoses presenting to other departments. This was similar to that of lesions seen in the GUM clinic, with $8(22 \%)$ patients with LSc, eight (22\%) with non specific dermatitis (NSD), six (17\%) with wart virus infection and one (3\%) with PIN seen in other departments.

The histological diagnoses of these biopsies are shown in table 3.

\section{Discussion}

There is now considerable interest in vulval cutaneous disorders, ${ }^{34}$ but relatively little 
Table 3 Diagnoses in patients undergoing penile biopsy in other departments

\begin{tabular}{lll}
\hline Department & Diagnosis & No. of Patients $(n=36)$ \\
\hline Dermatology & Non specific dermatitis & 1 \\
& PIN 3 & 1 \\
& Wart virus infection & 1 \\
General Surgery & Haemangioma & 1 \\
& Lichen sclerosus & 2 \\
Urology & Wart virus infection & 2 \\
& Non specific dermatitis & 7 \\
& Lichen sclerosus & 6 \\
& Normal (social circumcisions) & 5 \\
& Wart virus infection & 3 \\
& $2^{\circ}$ carcinoma of prostate & 1 \\
& $2^{\circ}$ carcinoma of bladder & 1 \\
& Cutaneous tuberculosis & 1 \\
& Epidermal cyst & 1 \\
& Squamous cell carcinoma & 1 \\
& Trauma & 1 \\
\hline
\end{tabular}

information is available about the cutaneous pathology of the penis. ${ }^{56}$

Penile biopsy is a procedure which is performed rarely in GUM and other clinics, in contrast to many other specialties where the value of diagnostic biopsy is now well established. Whilst reluctance on behalf of the clinician and the patient may be easily understood, we have found the procedure to be technically straight forward, well tolerated and safe. Considerable clinically useful information was obtained, with few complications, and a cosmetically acceptable end result.

The variety of skin types found in different parts of the penis may account, at least in part, for the diversity of cutaneous abnormalities seen in this area. However, as with skin elsewhere, penile skin can react only in a limited number of ways to endogenous and exogenous influences, so providing a restricted number of clinical presentations. It may be difficult, therefore, to diagnose conditions such as plasma cell balanitis and PIN without a biopsy.

It is currently unknown to what extent treatment alters the histological features of penile skin, and it might prove difficult to obtain ethical approval for such a study. However, given that all forms of treatment were withheld for a minimum of two weeks prior to biopsy, we felt that such effects had been reasonably excluded.

We postulate that the relatively fragile glabrous skin of the glans penis may be particularly sensitive to external irritants, thus explaining why NSD was found frequently in our study, and its predominant location in the preputial sac. The clinical course had typically been a relapsing and remitting one, usually helped transiently by the application of $1 \%$ clotrimazole (Canesten) cream. The most frequent pre-biopsy diagnosis, therefore, was fungal balanitis. However, such cases of "candidal" or "fungal" balanitis were found to have histological changes of NSD, rather than the characteristic finding associated with fungal colonisation. The pathological changes of NSD can be produced by a wide variety of clinical conditions, including partially treated infections. Clearly inadequate hygiene in uncircumcised men could cause changes of NSD, but the patients seen were notably conscientious about their penile hygiene. Although it is difficult to exclude completely a diagnosis of fungal infection, the majority of individuals had either a family or personal history of atopy, and it was considered that the most likely diagnosis was eczema. In these cases, clotrimazole cream may have been simply acting as an emollient or by some mechanism other than its antifungal activity. ${ }^{78}$

The malignant potential of $\mathrm{LSc}$ is currently unclear. In one study, ${ }^{9}$ LSc was found in 48 $(61 \%)$ of 78 women with vulval squamous carcinoma, either adjacent to the lesion or elsewhere on the body. There is less information on penile LSc, although three cases of penile squamous carcinoma have been reported in association with $\mathrm{LSc} ;{ }^{10}$ other reports are sporadic. ${ }^{112}$ The finding of LSc in biopsy specimens of genital cancer does not necessarily imply it is premalignant, particularly in view of its frequent occurrence in males circumcised for other reasons. ${ }^{13}$ However, in view of the current uncertainty about possible malignant development, long term monitoring would seem wise. ${ }^{14}$

Only $25 \%$ of cases of lichen planus (LP) were suspected prior to biopsy, and no men had any evidence of disease elsewhere. LP affects mucocutaneous membranes and exhibits characteristic histological features. ${ }^{1}$ Vulval lichen planus can be solitary and erosive, which may be difficult to diagnose on both clinical and pathological grounds. ${ }^{15}$ Our experience would suggest that this is also true of penile LP.

Current views on the aetiology are that LP is a cell mediated immune disorder, in which the primary antigen has not yet been identified. $^{1617}$ Oral LP has a malignant potential, and it has been recommended that vulval LP be followed up long term. ${ }^{15}$ Less is known of penile LP. There have been two case reports of LP found in association with PIN and verrucous carcinoma of the penis, ${ }^{18}$ so it could be argued that penile LP should also be monitored indefinitely.

The four cases of PIN seen in this study were all referred on the grounds that they had atypical appearances which were similar to sessile warts. The concept of PIN has recently developed as an extension of the ideas behind the classification of cervical intraepithelial neoplasia (CIN) and vulval intraepithelial neoplasia. The histological features are well characterised, ${ }^{2}$ although the clinical manifestations are less well reported. Like CIN, the malignant potential of PIN has still to be fully evaluated.

The range of histological diagnoses made in the other departments was broadly similar to that in the PDC. Men with penile dermatoses present to a variety of specialties, but each specialty may see only occasional cases. In view of the understandable concern of many patients that their penile dermatosis may have a sexually transmissible component, GUM physicians are ideally placed to exclude such diseases and provide any appropriate treatment and follow up. Our information indicates that, given the current lack of knowledge concerning the malignant potential of LSc, LP and 
PIN, 27\% (16 of 61 ) of patients attending the PDC and $33 \%$ of patients attending other departments may need long term review.

Information concerning the aetiology, natural history and treatment of penile dermatoses is sparse. Our data suggest that diagnoses based on clinical appearance alone are inadequate. The skin snip biopsy technique provides a simple, minimally invasive procedure which markedly improves the rate of diagnosis of such conditions. Pathological diagnoses were obtained in the majority of cases, thus allowing definitive treatment where indicated, and the chance to learn more in cases where our understanding is limited. It is often reassuring for the patient to know the diagnosis, even where current treatment options are limited.

1 Lever WF, Schaumberg-Lever G. Histopathology of the Skin. 7th ed. Philadelphia: JB Lippincott Company, 1990.

2 Barrasso $\mathrm{R}$, De Brux J, Croissant O, Orth G. High prevalence of papillomavirus-associated penile intraepithelial neoplasia in sexual partners of women with cervical intraepithelial neoplasia. $N$ Engl $f$ Med 1987;317: 916-23.

3 Ridley $\mathrm{CM}$. General dermatological conditions and dermatoses of the vulva. In: Ridley $\mathrm{CM}$ ed. The Vulva. Edinburgh: Churchill Livingstone, 1988:138-211.

4 Wilkinson EJ ed. Pathology of Vulva and Vagina. Contemporary Issues in Surgical Pathology. Vol. 9. New York: Churchill Issues in Surgical
Livingston, 1987.

5 Peterson RO. Penis. In: Peterson RO, ed. Urologic Pathology. Philadelphia: JB Lippincott Company, 1986:669-704.
6 Arumainayagam JT, Sumathipala AHT. Value of performing biopsies in genitourinary clinics. Genitourin Med 1990; 66:407

7 Holt RJ, Newman RL. Laboratory assessment of the antimycotic drug clotrimazole. $f$ Clin Pathol 1972;25: 1089-97.

8 Schaller $\mathrm{K}$. In vitro antibacterial activity of different clotrimazole formulations. Chemotherapy 1982; 28(Suppl.1):32-6.

9 Leibowitch M, Neill S, Pelisse M, Moyal-Baracco MM. The epithelial changes associated with squamous cell carcinoma of the vulva: a review of the clinical, histological and viral findings in 78 women. Br 7 Obstet Gynaecol viral findings in

10 Doré B, Irani J, Aubert J. Carcinoma of the penis in lichen sclerosus et atrophicus. Eur Urol 1990;18:153-5

11 Bart RS, Kopf AW. Squamous-cell carcinoma arising in balanitis xerotica obliterans. F Dermatol Surg Oncol 1978;4:556-7.

12 Bingham JS. Carcinoma of the penis developing in lichen sclerosus et atrophicus. Br f Venereal Dis 1978;54:350-1.

13 Ledwig PA, Weigand DA. Late circumcision and lichen sclerosus et atrophicus of the penis. $7 \mathrm{Am}$ Acad Dermatol 1989;20:211-4.

14 Ive FA, Wilkinson DS. Diseases of the umbilical, perianal and genital regions. In: Rook A, Wilkinson DS, Ebling FJG, Champion RH, Burton JL eds. Textbook of Dermatology. 4th ed. Edinburgh: Blackwell Scientific Publications, ogy. 4th ed. Edin.

15 Ridley CM. Chronic erosive vulval disease. Clin Exp Dermatol 1990;15:245-52.

16 Norris DA. Cytokine modulation of adhesion molecules in the regulation of immunologic cytotoxicity of epidermal targets. F Invest Dermatol 1990;65(6 Suppl): 111 S-120S.

17 Griffiths CE, Voorhees JJ, Nickoloff BJ. Characterisation of intracellular adhesion molecule-1 and HLA-DR expression in normal and inflamed skin: modulation by recombinant gamma interferon and tumour necrosis factor. $\mathcal{f}$ Am Acad Dermatol 1989;20:617-29.

18 Bain L, Geronemous R. The association of lichen planus of the penis with squamous cell carcinoma in situ and with the penis with squamous cell carcinoma in situ and with verrucous squamo
$1989 ; 15: 413-7$. 